\title{
EXPERIMENTAL STUDY ON BEARING RESISTANCE OF SHORT DISPLACEMENT PILE GROUPS IN DENSE SANDS
}

\author{
Arnoldas NORKUS ${ }^{1 *}$, Vaidas MARTINKUS ${ }^{2}$ \\ ${ }^{1}$ Laboratory of Geotechnics, Vilnius Gediminas Technical University, Vilnius, Lithuania \\ ${ }^{2}$ CSD Engineers, Vilnius, Lithuania
}

Received 26 March 2019; accepted 02 April 2019

\begin{abstract}
The prediction of the behavior of structures interacting with soil is one of the main challenges in structural design. Accurate evaluation of soil-structure interaction ensures a rational design solution for the superstructure and foundation of a building. In structural analysis, one of the key problems is the identification of relevant movements of the foundation considering the interaction between the superstructure, foundation and ground (the soil mass around the foundation). The correct assessment of soil-structure interaction contributes to the rational constructional design of the superstructure and foundation and allows avoiding violations of requirements for ultimate and serviceability limit states possible due to unpredicted additional stress on the structural system. Resistance predictions for pile group foundations is a complex problem, which may be the reason for scattered and insufficient information available despite numerous experimental and numerical studies, predominated by the focus on partial empirical relationships. This experimental study analyzed the prototype of a short displacement pile group with a flexible pile cap in terms of the bearing capacity and deformation behavior while subjected to static axial vertical load. In particular, attention was given to the resistance-stiffness evolution of single piles acting in a pile group with different spacing. Test results of short displacement pile groups were used to verify known models for the bearing resistance prediction of the pile group.
\end{abstract}

Keywords: displacement pile group, resistance, stiffness, spacing, sand, static vertical compressive load, density.

\section{Introduction}

A displacement pile (DP) is considered one of the most efficient foundation types because pile installation compacts granular soil increasing the load bearing resistance and stiffness of the ground (the soil mass around the pile). Short DPs apply when strong layers of soil are shallow.

Performance prediction methods for a single pile (SP) and pile group (PG) have long been the center of attention due to their relevance for geotechnical engineering. Investigations of the resistance performance of a single pile include experimental (Ai \& Yue, 2009), numerical (Sheng, Dieter, \& Wriggers, 2005; Said, De Gennaro, \& Frank, 2009) and combined (Yetginer, White, \& Bolton, 2006).

General aspects of ground resistance and PG deformation were analyzed by Adejumo (2013), Broms (1981), Mandolini and Viggiani (1997), Poulos (1968), Randolph (2003) and Vesic (1975). Experimental investigation results were presented by Al-Mhaidib (2006), Kishida (1964), Krasinski and Kusio (2014), McCabe and Lehane (2006), Randolph, Dolwin, and Beck (1994), Sales, Prez- zi, Salgado, Choi, and Lee (2017), Tejchman (1973), Tuan (2016), Vesic (1967, 1968), Q.-q. Zhang, S.-m. Zhang, Liang, Q. Zhang, and Xu (2015). Numerical simulation results were introduced by Bhasi, Rajagopal, and Reddy (2010), Choi, Lee, Prezzi, and Salgado (2017), Comodromos, Anagnostopoulos, and Georgiadis (2003), Gogoi, Sanandam and Binu (2014), Gowthaman and Nasvi (2018) and Ju (2013).

Despite the existing experimental and numerical studies, the currently proposed prediction methods yield rather scattered magnitudes of single pile and pile group resistances. The distribution is caused by numerous factors, such as survey methodology, pile types, testing equipment, installation type, slenderness ratio, relative density, soil type, pile-cap connection etc. Analytical and empirical models for the prediction of the ground resistance were developed for long piles (the slenderness ratio $L / D \geq 20$ ); therefore, the suitability of the proposed prediction methods for less slender (short) piles (which are frequently used in Lithuania $(L / D \approx 5-6))$ should be verified experimentally.

${ }^{\star}$ Corresponding author. E-mail: arnoldas.norkus@vgtu.lt 
The study aimed to conduct the static axial compression tests and perform a resistance analysis for a short single displacement pile (SDP) and a short displacement pile group (DPG), both installed in an artificially created sand deposit.

\section{Ground resistance of a pile and pile group}

Ground resistance of a single pile loading is determined in terms of stress distribution states under the pile base $\sigma_{b}$ and along the pile skin surface $\tau_{s}$ at all loading stages. The ultimate bearing capacity of a single pile is described via its ultimate load $Q_{u}$, corresponding to ground resistance at the deformation stage when the prescribed ultimate settlement $s_{u}$ is achieved. This conditional criterion of the ultimate limit state usually dominates in design. In terms of point forces, $Q_{u}$ and the effective weight load $W_{\text {pile }}^{\prime}$ of a submerged pile are equilibrated using the shaft resisting force $Q_{s u}$ and the base resisting force $Q_{b u}$ as follows:

$$
Q_{u}+W_{p i l e}^{\prime}=Q_{b u}+Q_{s u}=\sigma_{b} \cdot \frac{\pi \cdot D^{2}}{4}+\pi \cdot D \cdot \int_{0}^{L} \tau_{s} \cdot d z,
$$

where $L, D, \sigma_{b}$ and $\tau_{s}$ are the pile length, diameter, base bearing (base contact stress) resistance and shaft bearing (shaft contact stress) resistance, respectively. The maximum $\sigma_{b}$ is mobilized in the case of large pile settlement $s$, having the order of $5-10 \%$ of $D$. On the contrary, the maximum $\tau_{s}$ is mobilized at a much smaller $s$, having the order of $0.5-2 \%$ of $D$. The distribution of a pile shaft and base stresses for the considered limit state $\sigma_{b u}$ and $\tau_{s u}$ determine the appropriate ultimate stress state of the pile ground. As the pile base resistance and the shaft maximum resistance does not mobilize simultaneously, the ultimate pile bearing capacity corresponds to the ground stress state for fixed relative pile settlement $s_{u}$, mostly $10 \%$ of $D$, i. e. $s_{u}=0.1 D$ (European Committee for Standardization [CEN], 2004; Viggianni, Mandolini, \& Russo, 2012). Note that the distribution of $\sigma_{b}$ and $\tau_{s}$ for SDP is generally nonlinear at all stages of ground deformation and only their shape changes due to the development of pseudoelastic and plastic stresses and strains (Martinkus, Norkus, Statkus, \& Zilioniene, 2014). The evolution of stress-strain state during loading highly depends on initial residual stresses and the strain state, developed by the installation of SDPs separately and in DPG. Thus, the bearing resistance and the stiffness of the DPG foundation depend on a complex of peculiarities pertaining to the elastic-plastic stress-strain evolution of the ground resulting from the soil-pile interaction of SPs.

For practical purposes in geotechnical engineering, the evaluation of the total bearing resistance of a pile group (PG) is made by assessing the bearing resistances of isolated SPs. A primary concept of PG efficiency (the bearing capacity increment/reduction) was realized via an empirical factor $\eta$, a multiplier introduced for summing the SP bearing capacities. Kishida (1964) concluded, that $\eta$ in sands depends on (1) the pile spacing ratio $a / D$, where $a$ is the spacing of piles; (2) the PG configuration; and (3) the peak effective friction angle $\varphi_{p}$ '. He reported that in the case of $a / D=2$, the $\eta$ varies within bounds of 0.8 and 2.5. The larger magnitude was proposed for sand with $\varphi_{p}{ }^{\prime}=$ $30^{\circ}$ and the smaller magnitude for $\varphi_{p}{ }^{\prime}>45^{\circ}$. He stated that $\eta=1$ (i. e. independent of $\varphi_{p}{ }^{\prime}$ ) for $a / D \geq 6.5$. According to the analogous experimental findings by Vesic (1968), (1) $\eta$ is related only to $\tau_{s}$ of SPs, i. e. $\eta=\eta_{s}$; and (2) $\eta_{\max }=3$. Based on PG test results in loose and dense sands by Tejchman (1973), (1) $\eta \geq 1$ for various PG configurations; and (2) the reduction of $a$ increases the PG bearing capacity. Broms (1981) analyzed the variation of $\eta$ by performing PG tests with small scale piles made of different materials with rough and polished surfaces. His findings were in line with Kishida's (1964) but in conflict with the conclusions by Vesic (1968) and Tejchman (1973). Gogoi et al. (2014) tested groups of bored micropiles that were very slender using them in medium dense sand. They reported the tendency of the increment $\eta$ up to $a / D=4$, and beyond this limit, the decrease was related with the increase of $a$. Zhang et al. (2015) tested and numerically simulated the PGs connected by a rigid cap. According to their findings, (1) the settlement $s$ of PG decreases in the case of a smaller $a$ at the same loading level; (2) the increment $\eta$ is compatible with the increment $a$; and (3) $s$ of PG decreases with the increasing $a$ and with the growing number of piles. Tuan (2016) numerically simulated PGs with different spacings $a$ in granular soils of various densities and performed full-scale field tests. He stated that the stiffness of a pile cap increases $\eta$ and proposed the empiric formula for $\eta$ in relation with shaft and base resistances of SPs. Choi et al. (2017) performed an experimental study with model PGs driven in the sand of various densities. He stated that $\eta$ was slightly less than 1 for the PG driven in dense sand, while $\eta>1$ for the PG driven in medium dense and loose sand. Sales et al. (2017) tested PGs in sands of various densities. According to their findings, (1) the spacing $a$ in sand has minimal effect when $a / D>4 ;(2) \eta>1$ is for loose to medium dense sand, while $\eta<1$ in dense sand; and (3) the driving effects of SPs had an impact on the load-settlement response of PGs with spacings $a=2 D, 3 D$, whereas for $a=4 D$, installation effects were negligible. Gowthaman and Nasvi (2018) analyzed PG behavior in dilatant soils. Their findings state that (1) $\eta$ increases with $a$ up to $8 D$, their magnitudes approaching to 1 ; and (2) the stiffness of PG decreases when $a$ decreases. Sharma, Sushanta, and Zakir (2019) performed an experimental study in loose, medium dense and dense sands. They stated the positive group effect for loose to medium sands, and the negative group effect for dense sands (with the relative density of $80 \%)$. They summarized that the slenderness ratio $L / D$, the pile spacing, and the relative density were the most important factors for $\eta$ magnitude.

Fleming, Weltman, Randolph, and Elson (2009) proposed a concept of an equivalent raft for PG response analysis. The PG considered as a particular block of soil reinforced by piles with two available failure modes, namely, (1) failure of individual SPs; and (2) failure of the soil block. The following failure scenarios were forecasted: (1) 


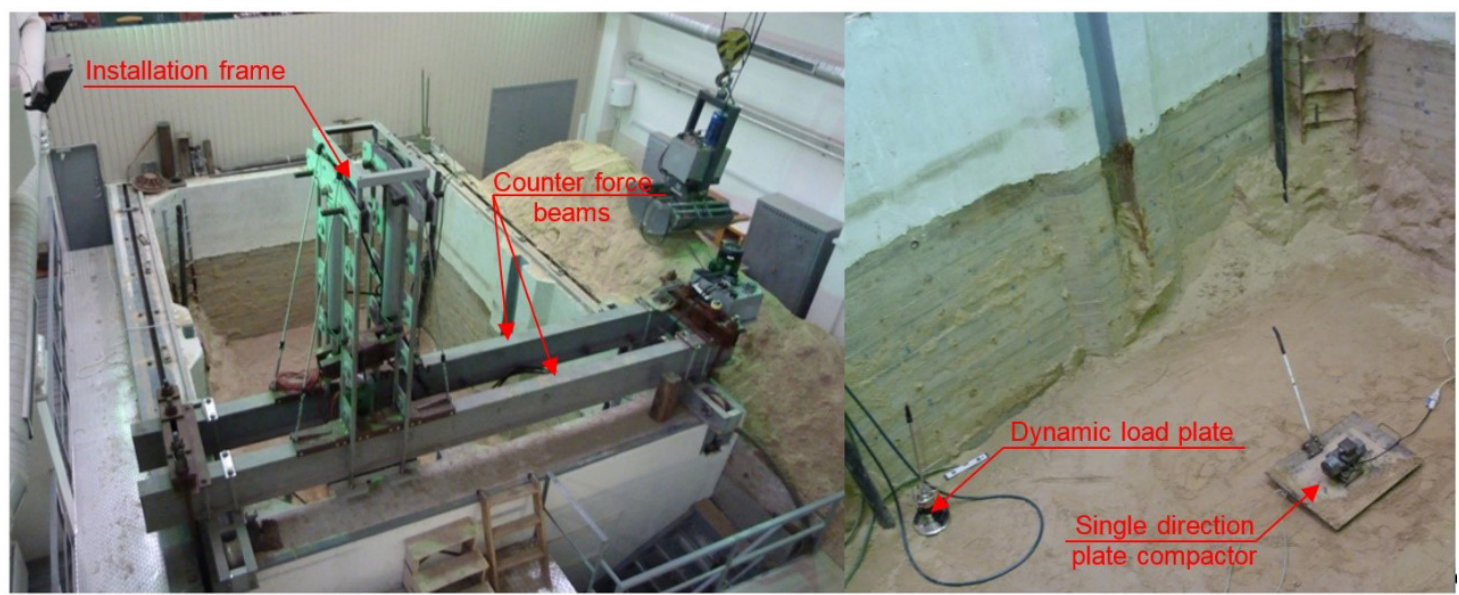

(a)

(b)

Figure 1. Equipment of the specialized soil testing box: (a) itinerant; (b) non itinerant

PG of a large number of long slender piles is more likely to fail as the block of soil when compared with the foundation of short stubby piles with the same spacing; and (2) the failure of the soil block in sand is less likely for piles placed in clay.

The settlement $s$ of PG foundation is larger than the sum of SP settlements for the same foundation load (Fleming et al., 2009; Terzaghi, 1943, etc.). For the prediction of $s$, Randolph (2003) developed the equivalent raft method, adopting the PG foundation as a massive rigid soil block. However, despite a long term of use, the method has significant shortcomings, such as ignoring significant factors, namely, reciprocal pile interaction, installation technology, pile cap stiffness, and uneven distribution of ground stiffness. Various investigations of numerical modelling of PG response are available (Bhasi et al., 2010; Ju, 2013; Comodromos et al., 2003; McCabe \& Lehane, 2006, etc.). A numerical simulation result is very sensitive not only in terms of the applied mathematical model but also its initial parameters. In this case, the sensitivity analysis of SP and PG performance predictions (e.g., Kala \& Vales, 2017; Chalmovsky et al., 2017; Stefanak, Kala, Mica, \& Norkus, 2018) can be useful. The settlement $s$ of an individual pile acting in PG is generally determined by using a superposition of interaction factors. In studies of Mylonakis and Gazetas (1998), Randolph and Wroth (1979), the interaction coefficient $\alpha$ was introduced. The coefficient defined a fractional $s$ increase of SP, resulting from the presence of loaded adjacent SPs. Hence, the method intended to evaluate the SP-soil interaction by quantifying the particular SP stiffness via its response measures. The method applies when the PG cap is relatively flexible. When PG cap is rigid, the condition of equal $s$ for all individual SPs acting in PG is satisfied.

All of the methods mentioned above, as well as other analytical, empirical, and numerical methods, propose techniques for the prediction of PG response measures, which were developed for solving actual practical problems. No factor of the SDP installation sequence for the prediction of their individual response measures and that for the DPG as a whole was found in the literature.

\section{Experimental set-up and ground characterization}

Pile tests were conducted under laboratory conditions in a specialized soil test box of $5.0 \mathrm{~m}$ in width, $7.0 \mathrm{~m}$ in length and $4.5 \mathrm{~m}$ in depth (Figure 1). First, a soil layer of $2.5 \mathrm{~m}$ in thickness was dug out (Figure 1(a)); then, the box was filled with compacting sand layers up to the initial level (Figure 1(b)).

The control of compaction was ensured by the Dynamic Plate Load (DPL) tests. 12 DPL tests were performed for each layer. Nine samplings (three samples from three points, Figure 2) were investigated to determine soil properties. The artificial deposit was described as even-graded medium-coarse (Figure 3) silica sand. 24 direct shear tests under the Constant Normal Load condition were carried

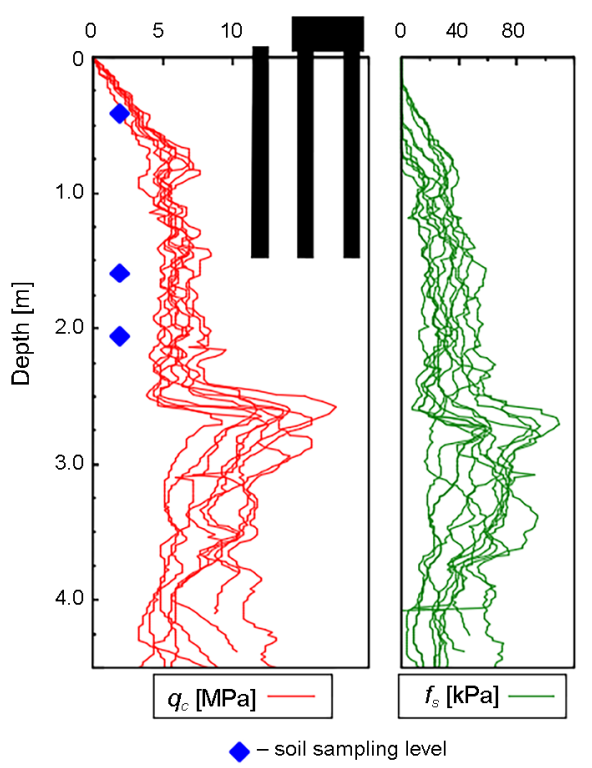

Figure 2. CPT data 


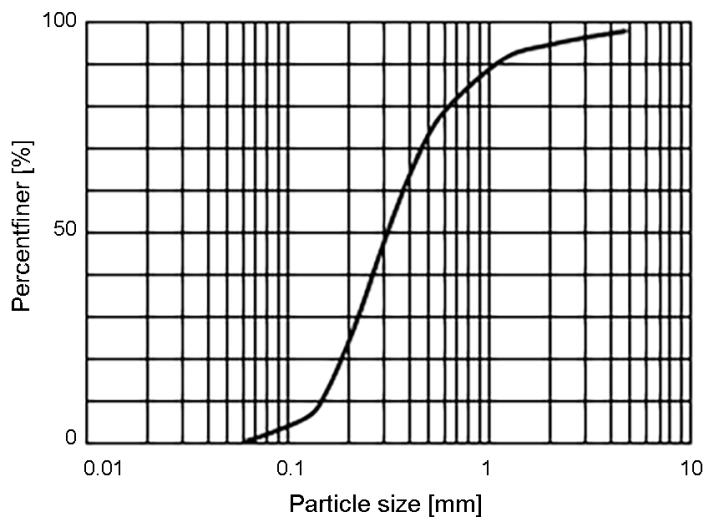

Figure 3. Curve of the particle size distribution

Table 1. Soil parameters

\begin{tabular}{|l|c|c|}
\hline \multicolumn{3}{|c|}{ Soil physical and mechanical parameters } \\
\hline Soil density $\rho$ & 1.64 & $\mathrm{~g} / \mathrm{cm}^{3}$ \\
\hline Soil moisture content $w$ & 4.38 & $\%$ \\
\hline Soil particle density $\rho_{s}$ & 2.65 & $\mathrm{~g} / \mathrm{cm}^{3}$ \\
\hline Mean particle size $d 50$ & 0.33 & $\mathrm{~mm}$ \\
\hline Void ratio $e$ & 0.69 & \\
\hline Maximal soil void ratio $e_{\max }$ & 1.65 & \\
\hline Minimal soil void ratio $e_{\min }$ & 0.44 & \\
\hline Relative density $D_{r}$ & 0.79 & \\
\hline Friction effective angle at critical state $\varphi_{c s}{ }^{\prime}$ & 30.5 & $\mathrm{o}$ \\
\hline Peak friction effective angle $\varphi_{p}{ }^{\prime}$ & 30.5 & ${ }^{\circ}$ \\
\hline Peak dilation angle $\psi_{p}{ }^{\prime}$ & 26 & ${ }^{\circ}$ \\
\hline Cohesion $c^{\prime}$ & 0 & $\mathrm{kPa}$ \\
\hline Odometer compressibility index $C_{c}$ & 24.62 & $\mathrm{MPa}$ \\
\hline Average DPL deformation modulus $E_{d}$ & 22.90 & $\mathrm{MPa}$ \\
\hline
\end{tabular}

out. Three odometer and three consolidated triaxial tests were performed. 12 Cone Penetration Tests (CPT) were carried out at the same locations of DPL tests (Figure 2).

Based on determined soil properties, the pile ground was dense sand. All soil properties are summarized in $\mathrm{Ta}$ ble 1.

\section{Pile and pile group testing equipment, installation and loading procedures}

Ground resistance measures were recorded by loading SDPs and DPGs connected by a cap. Steel pile prototypes Model Pile (MP) and Regular Pile (RP) were employed to perform tests (Figure 4). MP was originally constructed to determine normal stresses $\sigma_{b 1}$ and $\sigma_{b 2}$ on two particular areas $A_{b 1}$ and $A_{b 2}$ of the pile base and shear stresses $\tau_{s 1}$ and $\tau_{s 2}$ on two particular areas of the pile skin $A_{s 1}$ and $A_{s 2}$. The aforementioned areas were selected considering the distribution peculiarities of $\sigma_{b}$ and $\tau_{s}$. The peculiari-

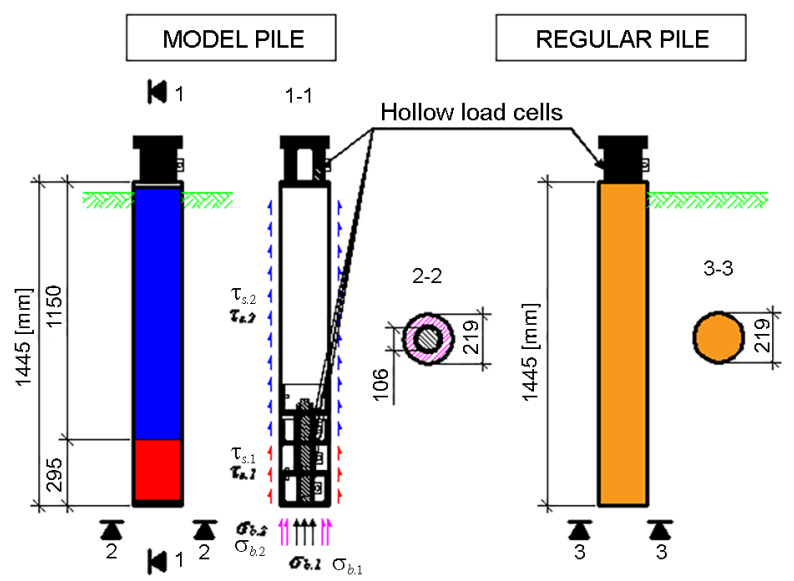

Figure 4. MP and RP schemes

ties were based on a strong correlation of base and radial (hence, shaft) stresses alongside the surface close to the pile base.

In the case of the RP, only the pile load $F$ was measured via the load cell placed on its head. The MP resisting force $F$ was calculated: $F=F_{b}+F_{s}=\left(F_{b 1}+F_{b 2}\right)+$ $\left(F_{s 1}+F_{s 2}\right)=\left(A_{b 1} \cdot \sigma_{b 1}+A_{b 2} \cdot \sigma_{b 2}\right)+\left(A_{s 1} \cdot \tau_{s 1}+A_{s 2} \cdot \tau_{s 2}\right) . \quad \mathrm{A}$ special constructional scheme for the analysis of ground resistance of an SP and DPG was designed and constructed. The steel beam grid (the cap analogue) transmitted the load to the DPG (Figure 5). Beams of the grid were flexibly connected. Beams and piles were connected using neoprene plates. From a hydraulic jack to beams, $F$ was transmitted via a steel spherical top. Thus, the load application and transmission to the SPs was ensured by a structural foundation scheme of hinged connections, conforming to the structural model of the DPG with a flexible cap.

Piles were penetrated in the soil applying the installation frame with two hydraulic cylinders (Figure 1(a)).

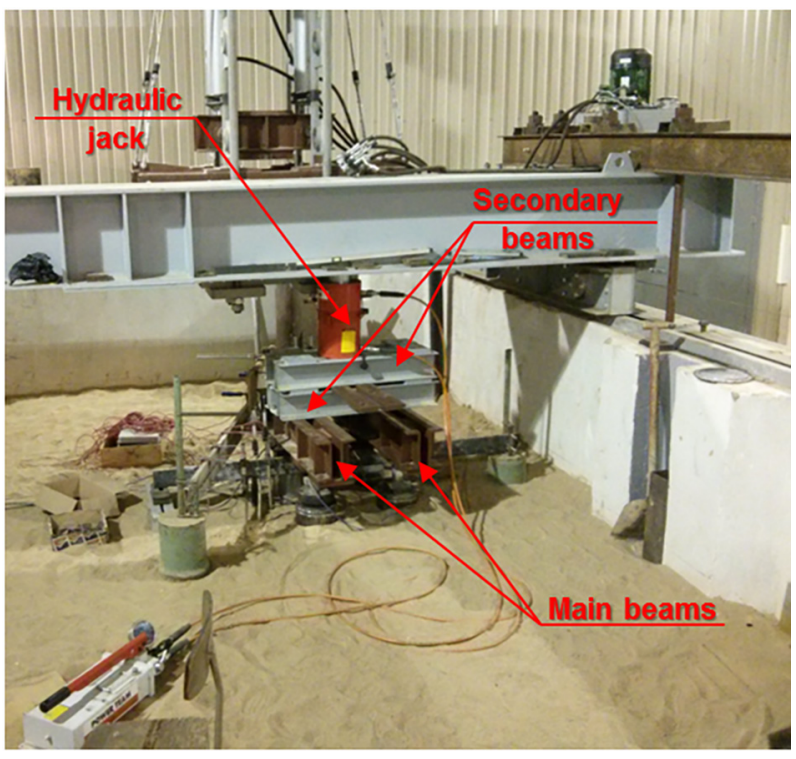

Figure 5. Equipment for testing the SDP and DPG 


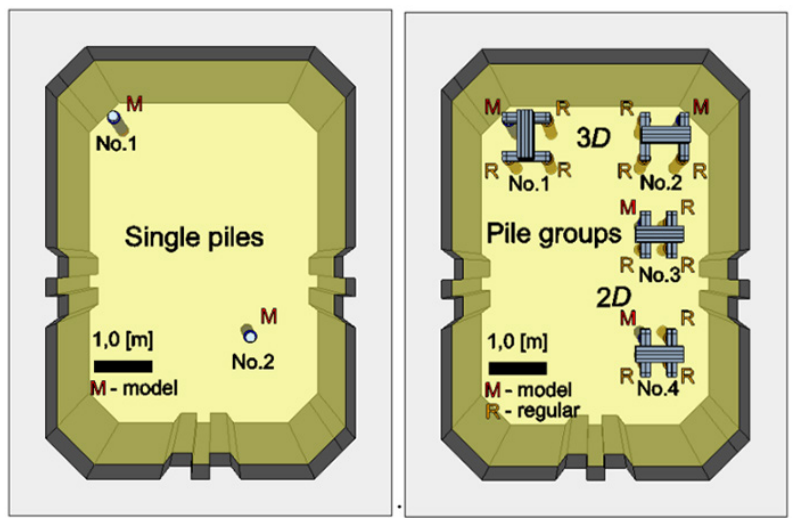

Figure 6. Location of analyzed SDPs and DPGs

Pushing velocity of the hydraulic cylinders was limited to $5 \mathrm{~mm} / \mathrm{s}$. The MP in all DPGs were installed first, and RPs were installed second, in the clockwise order for the PGs No. 1, No. 3 and No. 4, and in the anticlockwise order for the PG No. 2 (Figure 6). Aiming to investigate the effect of adjacent individual piles to the resistance of the MP acting in the DPG, the MP was tested twice; for the first time as an SDP, before installing the adjacent piles, and for the second time, once the adjacent piles have already been installed.

Piles were tested according to the standard ISO 22477 1:2018 (International Organization for Standardization [ISO], 2018). For $s_{u}=0.1 D$, the criterion of the ultimate limit state of a DP was used. The same criterion was used for the DPG. The evolution of the SDP and DPG resistances was analyzed within this interval of loading. As deformation of short steel piles was negligible compared with ground deformation, $s$ was equal to the total vertical displacement of the DPG's pile head.

\section{Analysis and discussion of results}

Aiming to reduce the amount of processed data, the averaging technique was applied to illustrate ground resistances of the SPs Nos. 1 and 2; DPGs with spacing $a=3 D$ of Nos. 1 and 2, and DPGs with spacing $a=2 D$ of Nos. 3 and 4 (Figure 6).

\subsection{Single pile tests}

The maximum ground resistance force $F$ was reached at $s_{u}=0.1 D$ (Figure 7 ). The base resisting force $F_{b}=$ $117 \mathrm{kN}$ contributed $76 \%$ to $F$. Consequently, the total shaft resistance force $F_{s}=37 \mathrm{kN}$ contributed $24 \%$ to $F$. The $\tau_{s}$ in the area near the pile base accumulated the force $F_{s, 1}$, the $7 \%$ of $F$, and $\tau_{s}$ in the upper part of the side surface $F_{s, 2}, 17 \%$ of $F$. The ratio $F_{b} / F_{s}=3.2$ determines that the tested DPs were base (tip) bearing piles.

\subsection{Tests for the pile groups with the $3 \mathrm{D}$ spacing}

The spacing $a=3 D$ is considered rational and is generally recommended for the design of PG foundations. The tested two DPGs consisted of symmetrically located SPs

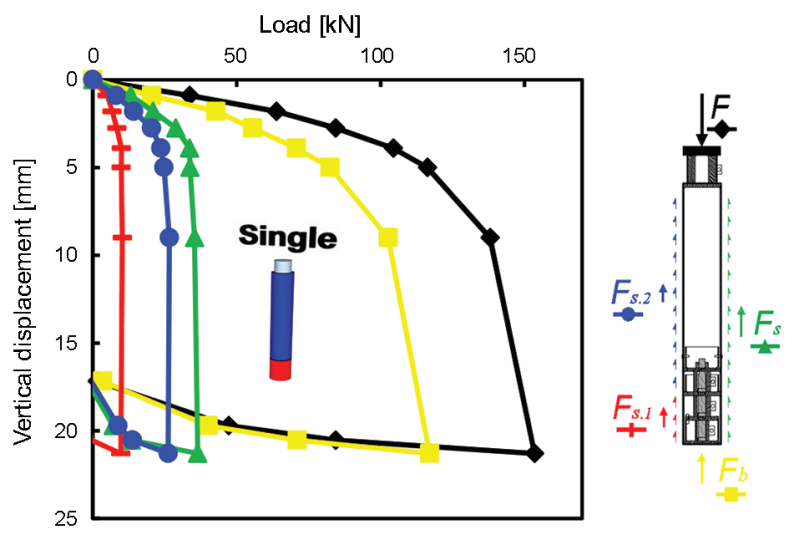

Figure 7. Averaged test graphs separate resisting forces $F_{i}$ vs settlement $s$ of an SDP

connected by a flexible cap. The $F$ vs $s$ graphs of SDPs acting in a DPG and that of the total DPG are presented in Figure 8. Note that settlements $s$ of individual SDPs significantly differed as the flexible cap did not restrict the distribution of the total load to SDPs proportionally to their actual individual stiffnesses. The DPG maximum load $F_{3 D \text {,max, group }}=640 \mathrm{kN}$ was at $s_{u}=0.1 D$. According to Figure 8, resistances of SDPs differ. Secant stiffness (the ratio of $F_{3 D \text {, max group }}$ to $s=0.1 D$ ) of separate SDPs varied within the bounds of $4910 \mathrm{kN} / \mathrm{m}^{\prime}$ and $9190 \mathrm{kN} / \mathrm{m}^{\prime}$. The ratio of the largest and the smallest secant stiffnesses was 1.87; the ratio of the average and the smallest stiffnesses was 1.4; and the ratio of the largest and the average stiffnesses was 1.33. Smaller secant stiffness magnitudes were characteristic to SDPs that were installed first and the larger magnitudes were particular to the piles installed later, which suggests that ground stiffness distribution under SDPs in the DPG depends on the installation sequence. Ground resistance of an MP acting in the DPG, in terms of the resulting forces is demonstrated in Figure 9. The maximum total load $F_{3 D, \text { max }}=141 \mathrm{kN}$. The $F_{b, 3 D}=108$ $\mathrm{kN}\left(77 \%\right.$ of $\left.F_{3 D, \text { max }}\right)$. The $F_{s, 3 D}=33 \mathrm{kN}\left(23 \%\right.$ of $\left.F_{3 D \text {, max }}\right)$. The contribution of the shaft resistance near the pile tip $F_{s, 1}$ was $5 \%$ of $F_{3 D \text {, max }}$ and the contribution of the mobilized resistance in the upper surface $F_{s, 2}$ was $18 \%$ of $F_{3 D \text {,max }}$.

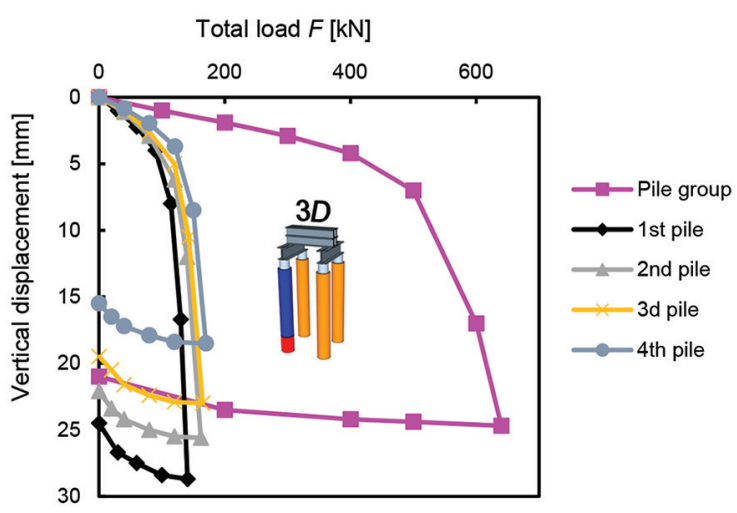

Figure 8. Averaged load $F$ vs $s$ graph of the DPG with the spacing $a=3 D$ 


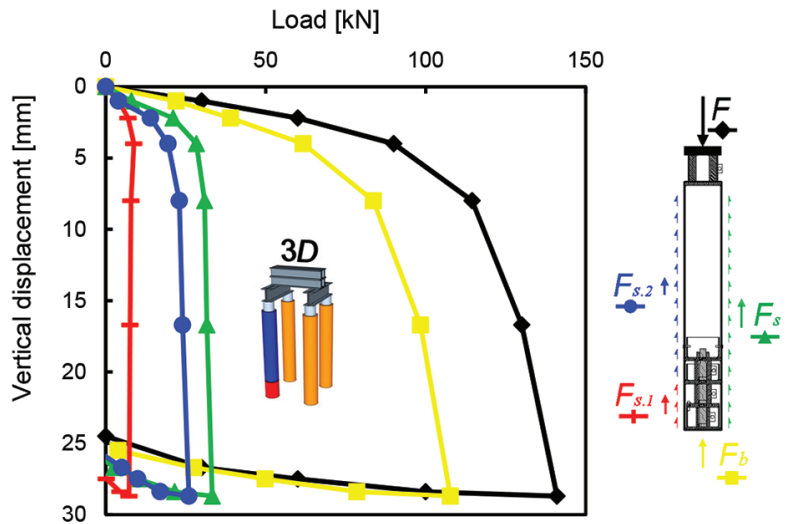

Figure 9. Averaged $F_{i}$ vs $s$ graph for the MP acting in the DPG with the spacing $a=3 D$

\subsection{Tests for the pile groups with the $2 \mathrm{D}$ spacing}

The $F$ vs $s$ graph of the DPG with the spacing $a=2 D$ (Figure 10) shows that the sum of four piles $F_{2 D \text { max, group }}$ is equal to $700 \mathrm{kN}$ at $s_{u}=0.1 D$. The stiffness (calculated in the same way as for the DPG with $a=3 D$ ) of each SDP varied from 5740 up to $14335 \mathrm{kN} / \mathrm{m}$ '. The ratio of the highest and the lowest stiffnesses was equal to 2.50 , the ratio of the average and the lowest stiffnesses was 1.55 ,

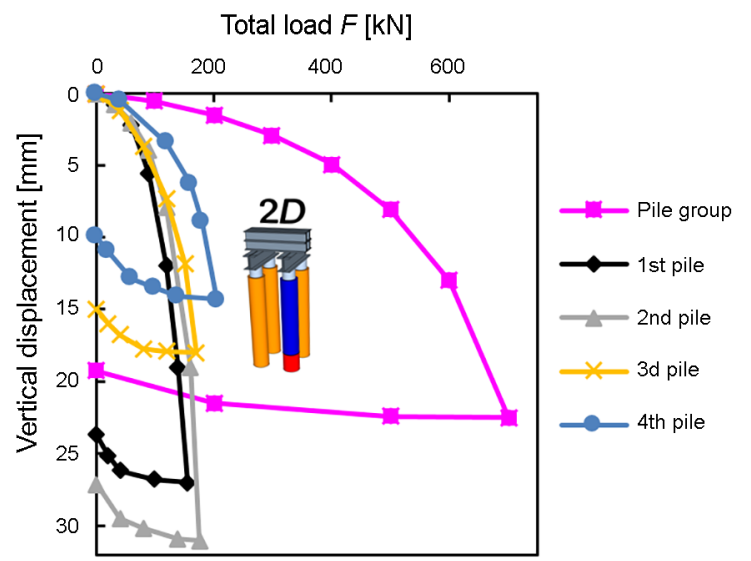

Figure 10. Averaged $F$ vs $s$ graph of the DPG tests with spacing $a=2 D$

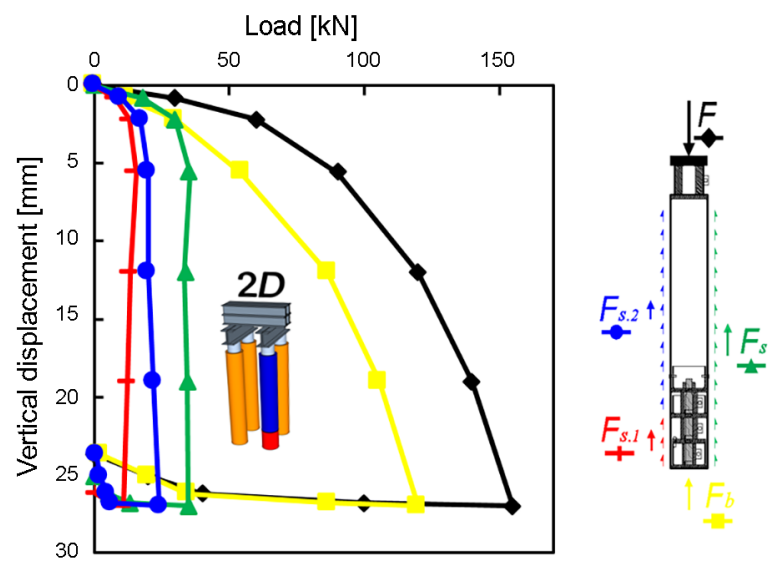

Figure 11. Averaged $F_{i}$ vs $s$ graph for the MP acting in the DPG with the spacing $a=2 D$ and the ratio of the average and the highest stiffnesses was 1.63. Thus, the stiffness distribution corresponds to the installation sequence, which is analogous to the DPG of $a=3 D$.

The performance of the MP in the DPG is shown in Figure 11. The total load $F=155 \mathrm{kN}$. The base resistance $F_{b}$ consisted of $77 \%$ of the total load $F=120 \mathrm{kN}$. The total shaft resisting force $F_{s}$ consisted of $23 \%$ of the total load $F_{2 D \text {, } \max }=35 \mathrm{kN}$. The shaft resistance $F_{s, 1}$ near the pile tip contributed $7 \%$ to the total load $F$ and the resistance of the upper part of the side surface $F_{s, 2}$ contributed $16 \%$ to the total load $F$.

\subsection{D spacing versus $2 \mathrm{D}$ spacing for the displacement pile group}

The bearing resistance vs $s$ peculiarities of DPGs with spacings $a=3 D$ and $a=2 D$ can be determined from their $F$ vs $s$ graphs comparing them with the "fictitious" DPG, created by applying the multiplier of the magnitude 4 for the analogous SDP graph (Figure 7). All graphs are presented in Figure 12. The DPG response character during loading stages was different. At early stages, $s=0.03 D$, the "fictitious" DPG resistance was the largest and decreased for the DPG with a smaller spacing $a$, namely, $480 \mathrm{kN}(0 \%)$; $465 \mathrm{kN}(-3 \%) ; 430 \mathrm{kN}(-12 \%)$ for the "fictitious" DPG as well as those with the spacings $a=3 D$ and $a=2 D$, respectively. At $s_{u}=0.1 D$, the result was the opposite: $616 \mathrm{kN}$ (0\%); $640 \mathrm{kN}$ (4\%); $700 \mathrm{kN}$ (14\%). These changes can be explained by the increment of the base resistance and the related shaft resistance near the pile tip (Figures 9 and 11). The significant increase in the shearing stress near the pile tip was observed for the DPG with $a=2 D$.

According to Figure 12, the behavior of the DPG with the spacing $a=3 D$ is more similar to the SDP behavior, and the DPG with the spacing $a=2 D$ is more similar to the behavior of a shallow foundation (soil block) as characterized by a more uniform performance. The analysis of the maximum and minimum stiffnesses of PDGs (Figures 8 and 10) demonstrates that the uniformity of the maximum and minimum secant soil-pile stiffnesses is up to $77 \%$ higher for the DPG with $a=2 D$.

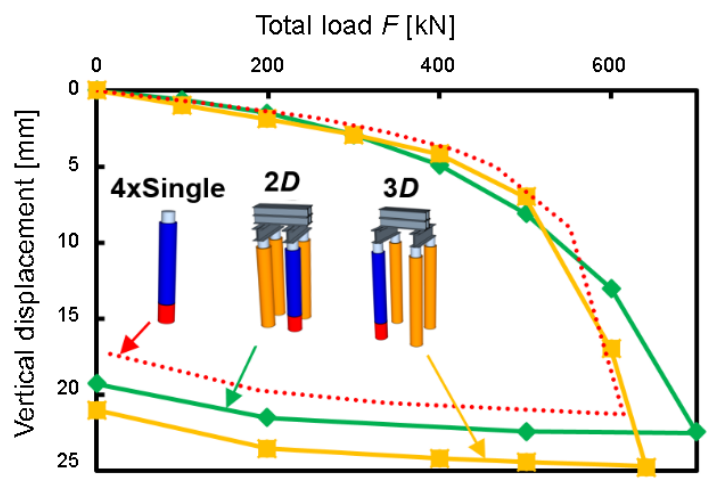

Figure 12. Averaged $F$ vs $s$ graphs of DPGs with the spacings $2 D$ and $3 D$ and the "fictitious" pile group created from averaged $F$ vs $s$ graph for an SDP 


\section{Concluding remarks}

The DPG efficiency factor $\eta$ for loading up to $s<0.1 D$ is in good agreement with the predictions by Vesic (1967, 1968) and Tejchman (1973) but is in conflict with the results determined by Broms (1981), Kishida (1964), Choi et al. (2017), Sales et al. (2017), Gowthaman and Nasvi (2018), Sharma et al. (2019), yielding $\eta<1$. Tests determined an increase of $\eta$ when decreasing the spacing $a$ in dense sands, and this result conflicts with the results reported by Gogoi et al. (2014) and Gowthaman and Nasvi (2018).

The significant increase of the shear stress near the pile tip and in the soil (inside the pile group) was observed for the DPG with $a=2 D$. This phenomenon shows the formation of the conditional block and the subsequent failure when increasing the load, which according to Fleming et al. (2009) is the phenomenon exceptionally particular to clays. The current finding is conditioned by the shape of the load vs settlement curves; the result that is more similar to the response of a shallow foundation than the behavior of a single displacement pile.

The significant difference of pile response in terms of stiffness subjected to the installation sequence was determined. The maximum and minimum stiffnesses of the DPG with $a=2 D$ differed by up to 2.5 times. The stiffness of an individual pile in the DPG with $a=2 D$ differed by up to $154 \%$. This result conflicts with the findings by Sales et al. (2017), stating the installation effects as negligible. From a practical point of view, this phenomenon might cause a rotation of the supported structure, consequently inducing additional internal forces, which should lead to the underestimation of the structure's stiffness and bearing capacity. This effect should be considered in the design of the constructional system as a whole consisting of the superstructure, foundation and ground.

The SDP response changed in principle for DPGs with the spacings $a=2 D$ and $a=3 D$ under different settlements. Small (up to $s=0.03 D$ ) settlements were characteristic to serviceability requirements for the superstructure. Within this $s$ interval, the DPG stiffness reduced when the spacing $a$ reduced. The contrary result was obtained when $s_{u} \geq 0.1 D$, i. e. for the settlements characteristic to the ultimate limit state requirements of the foundation. The DPG stiffness increased when the spacing $a$ reduced; it was also stiffer in the case of the "fictitious" DPG, created of separate SDPs. This result was in agreement with the findings by Zhang et al. (2015).

The obtained test results for the DPS and DPGs once again showed that the currently proposed methods for predicting pile group behavior measures are not only scattered but can even be in conflict. The sequence of DP installation was found to be an important factor for predicting the DPG efficiency and response measures. The effect should be properly evaluated in the design process together with the already recognized factors for the DPG resistance measures, such as slenderness, pile spacing, relative density and cap stiffness. The results of the performed ex- perimental study proved the necessity to specialize complementary tests with the aim to develop methods for the DPG behavior prediction, also applying numerical modelling techniques in concert with the validation and calibration procedures with test results.

\section{Disclosure statement}

Authors have not any competing financial, professional, or personal interests from other parties.

\section{References}

Adejumo, T. W. (2013). Analyses of behaviour of pile groups in layered clay. International Journal of Remote Sensing \& Geoscience (IJRSG), 2(2), 42-48.

Ai, Z. Y., \& Yue, Z. Q. (2009). Elastic analysis of axially loaded single pile in multilayered soils. International Journal of Engineering Science, 47(11-12), 1079-1088.

https://doi.org/10.1016/j.ijengsci.2008.07.005

Al-Mhaidib, A. I. (2006). Experimental investigation of the behaviour of pile groups in sand under different loading rates. Geotechnical and Geological Engineering, 24, 889-902. https://doi.org/10.1007/s10706-005-7466-8

Bhasi, A., Rajagopal, K., \& Reddy, V. (2010). Finite element study of the influence of pile jetting on load capacity of adjacent piles. International Journal of Geotechnical Engineering, 4(3), 361-370. https://doi.org/10.3328/IJGE.2010.04.03.361-370

Broms, B. B. (1981). Pre-cast piling practice. Thomas Telford.

Chalmovsky, J., Stefanak, J., Mica, L., Kala, Z., Skuodis, S., Norkus, A., \& Zilioniene, D. (2017). Statistical-numerical analysis for pullout tests of ground anchors. The Baltic Journal of Road and Bridge Engineering, 12(3), 145-153. https://doi.org/10.3846/bjrbe.2017.17

Choi, Y. S., Lee, J., Prezzi, M., \& Salgado, R. (2017). Response of pile groups driven in sand subjected to combined loads. Geotechnical and Geological Engineering, 35(4), 1587-1604. https://doi.org/10.1007/s10706-017-0194-z

Comodromos, E. M., Anagnostopoulos, C. T., \& Georgiadis, M. K. (2003). Numerical assessment of axial pile group response based on load test. Computers and Geotechnics, 30(6), 505-515. https://doi.org/10.1016/S0266-352X(03)00017-X

European Committee for Standardization (CEN). (2004). Eurocode 7: Geotechnical design - Part 1: General rules (EN 19971:2004).

Fleming, K., Weltman, A., Randolph, M., \& Elson, K. (2009). Piling engineering. Abingdon: Taylor \& Francis.

Gogoi, N., Sanandam, B., \& Binu, S. (2014). A model study of micropile group efficiency under axial loading condition. International Journal of Civil Engineering Research, 5(4), 323-332.

Gowthaman, S., \& Nasvi, M. C. M. (2018). Three-dimensional numerical simulation and validation of load-settlement behaviour of a pile group under compressive loading. Engineer: Journal of the Institution of Engineers, 51(1), 9-21. https://doi.org/10.4038/engineer.v51i1.7283

International Organization for Standardization (ISO). (2018). Geotechnical investigation and testing - Testing of geotechnical structures - Part 1: Pile load test by static axially loaded compression (ISO 22477-1).

Ju, J. (2013). Prediction of the settlement for the vertically loaded pile group using 3D finite element analyses. Marine GeoResources \& Geotechnology, 33(3), 264-271. https://doi.org/10.1080/1064119X.2013.869285 
Kala, Z., \& Vales, J. (2017). Sensitivity assessment and lateraltorsional buckling design of I-beams using solid finite elements. Journal of Constructional Steel Research, 139, 110-122. https://doi.org/10.1016/j.jcsr.2017.09.014

Kishida, H. (1964). The bearing capacity of pile groups under central and eccentric loads in sands (Report No. 19). Building research institute, Ministry of construction, Japanese Government.

Krasinski, A., \& Kusio, T. (2014). Comparative model tests of SDP and CFA pile groups in non-cohesive soil. Studia Geotechnica et Mechanica, 36(4), 7-11. https://doi.org/10.2478/sgem-2014-0031

Mandolini, A., \& Viggiani, C. (1997). Settlement of piled foundations. Géotechnique, 47(3), 791-816. https://doi.org/10.1680/geot.1997.47.4.791

Martinkus, V., Norkus, A., Statkus, T., \& Zilioniene, D. (2014). Experimental investigation of stresses in sand during the installation and loading of the short displacement pile. The Baltic Journal of Road and Bridge Engineering, 9(1), 10-16. https://doi.org/10.3846/bjrbe.2014.02

McCabe, B. A., \& Lehane, B. M. (2006). Behaviour of axially loaded pile groups driven in clayey silt. Journal of Geotechnical and Geoenvironmental Engineering, 132(3), 401-410. https://doi.org/10.1061/(ASCE)1090-0241(2006)132:3(401)

Mylonakis, G., \& Gazetas, G. (1998). Settlement and additional internal forces of grouped piles in layered soil. Geotechnique, 8(1), 55-72. https://doi.org/10.1680/geot.1998.48.1.55

Poulos, H. G. (1968). Analysis of the settlement of pile groups. Geotechnical Journal, 4, 449-471. https://doi.org/10.1680/geot.1968.18.4.449

Randolph, M. F. (2003). Science and empiricism in pile foundation design. Geotechnique, 53(10), 847-875. https://doi.org/10.1680/geot.2003.53.10.847

Randolph, M. F., \& Wroth, C. P. (1979). An analysis of the vertical deformation of pile groups. Geotechnique, 29(4), 423-439. https://doi.org/10.1680/geot.1979.29.4.423

Randolph, M. F., Dolwin, J., \& Beck, R. (1994). Design of driven piles in sand. Geotechnique, 44(3), 427-448. https://doi.org/10.1680/geot.1994.44.3.427

Said, I., De Gennaro, V., \& Frank, R. (2009). Axisymmetric finite element analysis of pile loading tests. Computers and Geotechnics, 36(1), 6-19. https://doi.org/10.1016/j.compgeo.2008.02.011

Sales, M. M., Prezzi, M., Salgado, R., Choi, Y. S., \& Lee, J. (2017). Load-settlement behaviour of model pile groups in sand under vertical load. Journal of Civil Engineering and Management, 23(8), 1148-1163. https://doi.org/10.3846/13923730.2017.1396559

Sharma, B., Sushanta, S., \& Zakir, H. (2019). A study of parameters influencing efficiency of micropile groups. In Ground Improvement Techniques and Geosynthetics (pp. 11-18). Singapore: Springer. https://doi.org/10.1007/978-981-13-0559-7_2

Sheng, E., Dieter, E. K., \& Wriggers, P. (2005). Finite element analysis of pile installation using large-slip frictional contact. Computers \& Geotechnics, 32(1), 17-26. https://doi.org/10.1016/j.compgeo.2004.10.004

Stefanak, J., Kala, Z., Mica, L., \& Norkus, A. (2018). Global sensitivity analysis for transformation of Hoek-Brown failure criterion for rock mass. Journal of Civil Engineering and Management, 24(5), 390-398. https://doi.org/10.3846/jcem.2018.5194
Tejchman, A. F. (1973). Model investigation of pile group in sand. Journal of Soil Mechanics and Foundation Division, 2, 99-215.

Terzaghi, K. (1943). Theoretical soil mechanics. London: John Wiley and Sons. https://doi.org/10.1002/9780470172766

Tuan, P. A. (2016). A simplified formular for analysis group efficiency of piles in granular soil. International Journal of Scientific \& Engineering Research, 7(7), 15-21.

Vesic, A. S. (1967). Ultimate loads and settlements of deep foundations in sand. In Proceedings of Symposium held at Duke University (pp. 53-68).

Vesic, A. S. (1968). Experiments with instrumented pile group in sand. In Proceedings of Seventy First Annual Symposium in Performances of Deep Foundations (pp. 177-222). https://doi.org/10.1520/STP47286S

Vesic, A. S. (1975). Principles of pile foundation design. Durham: Duke University.

Viggianni, C., Mandolini, A., \& Russo, G. (2012). Pile and pile foundations. London \& New York: Tailor \& Francis. https://doi.org/10.1201/b17768

Yetginer, A. G., White, D. J., \& Bolton, M. D. (2006). Field measurements of the stiffness of jacked piles and pile groups. Geotechnique, 56(5), 349-354. https://doi.org/10.1680/geot.2006.56.5.349

Zhang, Q.-q., Zhang, S.-m., Liang, F.-y., Zhang, Q., \& Xu, F. (2015). Some observations of the influence factors on the response of pile groups. KSCE Journal of Civil Engineering, 19(6), 1667-1674. https://doi.org/10.1007/s12205-014-1550-7

\section{Notations}

$$
\begin{aligned}
& \text { DP - displacement pile; } \\
& \text { SP - single pile; } \\
& \text { SDP - single displacement pile; } \\
& \text { PG - pile group; } \\
& \text { DPG - displacement pile group; } \\
& \text { MP - model pile; } \\
& \text { RP - regular pile; } \\
& L \quad \text { - pile length; } \\
& D \text { - pile diameter; } \\
& s \quad-\text { pile or pile group settlement; } \\
& s_{u} \quad \text { - pile prescribed settlement at limit state } \\
& (s=0.1 D) \text {; } \\
& \sigma_{b} \quad \text { - pile base bearing resistance (normal contact } \\
& F \quad \text { - load applied to pile or pile group; } \\
& F_{b} \quad \text { - base resisting force of pile; } \\
& F_{s} \quad \text { - shaft resisting force of pile; } \\
& \eta \quad \text { - pile group efficiency (bearing capacity) } \\
& \text { empirical factor; } \\
& \text { a - spacing of piles. }
\end{aligned}
$$

